Z Gerontol Geriat 2017 · 50:1-3 DOI 10.1007/s00391-016-1167-1

Online publiziert: 9 . Januar 2017

(c) Springer-Verlag Berlin Heidelberg 2017

CrossMark

Tania Zieschang ${ }^{2}$ Jürgen M. Bauer ${ }^{1,2}$

' Lehrstuhl für Geriatrie, Ruprecht-Karls-Universität, Heidelberg, Deutschland

${ }^{2}$ Agaplesion Bethanien Krankenhaus, Heidelberg, Deutschland

\title{
Menschen und Demenz
}

\section{Wie begegnen wir den Bedürfnissen der Betroffenen und denen ihrer Angehörigen?}

Forschungsfeld dar, in dem u. a. der regulatorischen Funktion der Myokine eine wesentliche Bedeutung zuzukommen scheint. Es ist naheliegend, für die Prävention des kognitiven Abbaus im Alter auch mehrdimensionale Ansätze $\mathrm{zu}$ verfolgen. Die in diesem Kontext während der letzten Jahre wichtigste Arbeit stellt die 2015 in Lancet veröffentlichte Finnish Geriatric Intervention Study to Prevent Cognitive Impairment and Disability (FINGER, [10]) dar. Hier wurden ältere Personen im Alter zwischen 60 und 77 Jahren, die ein erhöhtes Demenzrisiko aufwiesen, einer multidimensionalen Intervention zugeführt, die eine Ernährungsberatung, ein körperliches und kognitives Training sowie das Monitoring kardiovaskulärer Risikofaktoren einschloss. Positive Effektive auf das kognitive Leistungsvermögen zeigten sich im Vergleich zur Kontrollgruppe bereits nach 2 Jahren.

Von ebenso großer Bedeutung wie diese präventiven Ansätze sind jedoch auch der Umgang mit Menschen mit manifester Demenz und, wie weit es uns gelingt, auf ihre Bedürfnisse sowohl im ambulanten Setting, in den stationären Pflegeinrichtungen und im Krankenhaus einzugehen. Hier sind nicht nur die Gesundheitsberufe gefordert, sondern ebenso die Gesellschaft als Ganzes. Gemäß der UN-Behindertenrechtskonvention, die auch die Bundesregierung unterschrieben hat, haben alle Menschen mit Beeinträchtigung das Recht, am normalen gesellschaftlichen Leben teilzunehmen. In diesem Sinne ist es sehr zu begrüßen, dass man sich vielerorts auf den Weg zur demenzfreundlichen Kommune gemacht haben (Aktion Demenz e. V.: http://www.demenzfreundlichekommunen.de). Ebenso bedeutsam sind die wachsende Zahl an Sportangeboten für Menschen mit Demenz sowie die spezifischen Schulungsangebote für Trainer und Physiotherapeuten. Von einem flächendeckenden, niederschwelligen Angebot ist man in Deutschland jedoch noch weit entfernt. Dabei hat jedes Angebot, das die Selbstständigkeit und die Mobilität von Menschen mit Demenz fördert, das Potenzial, Kosten für die Solidargemeinschaft zu reduzieren, indem ein erhöhter Pflegebedarf, eine Institutionalisierung und der Ausfall von pflegenden Angehörigen am Arbeitsplatz vermieden werden.

Das vorliegende Schwerpunktheft der Zeitschrift für Gerontologie und Geriatrie fasst Einreichungen der letzten Monate zusammen und widmet sich in besonderem Maß der Versorgung von Patienten mit Demenz. Etwa drei Viertel der Menschen mit Demenz leben im eigenen Zuhause und werden dort von Angehörigen und/oder von ambulanten Pflegediensten unterstützt. Die hohe Belastung pflegender Angehörigen, die mit einem erhöhten Risiko für eine Depression, eine Verschlechterung des Gesundheitszustandes und für eine soziale Isolation einhergeht, ist bekannt [11]. Thyrian et al. beschreiben, dass die Belastung der Angehörigen nur unzureichend standardisiert erfasst wird und in diesem Kontext meist nur Screeninginstrumente verwendet werden. Im Rahmen ihrer Studie führten sie in Mecklenburg-Vorpommern eine ausführliche Erfassung der Belastung pflegender Angehöriger mithilfe des Berliner Inventar zur Angehörigenbelastung - Demenz (BIZA-D) ten Mechanismen stellt ein spannendes 
durch. Ihre Ergebnisse zeigen, wie stark die Belastung von spezifischen Merkmalen sowohl des betroffenen Patienten mit Demenz als auch des pflegenden Angehörigen abhängig ist. Die Autoren betonen, dass unterstützende Maßnahmen somit für den Einzelfall angepasst werden sollten und sich der diesbezügliche Bedarf im Verlauf der demenziellen Erkrankung verändern kann. Riedel et al. untersuchten die Nutzung von Selbsthilfegruppen durch pflegende Angehörigen von Menschen mit einer Alzheimer-Demenz. Unterstützung durch eine Selbsthilfegruppe nahmen in erster Linie pflegende Ehefrauen in den Städten wahr, wenn die Pflege durch starke funktionelle Einschränkungen sowie durch Verhaltensauffälligkeiten wie verbale oder physische Aggression erschwert wurde. Die Zufriedenheit mit den Selbsthilfegruppen war hoch. Als Gesellschaft sollten wir uns daher mit der Frage beschäftigen, wie niederschwellige Angebote auch im ländlichen Raum zu realisieren sind und wie Angebote für pflegende (Schwieger-) Töchter, die zusätzlich mit Berufstätigkeit und Kindererziehung betraut sind, aussehen sollten. In den letzten Jahren wird das Thema Demenz zunehmend auch in den Kommunen und im ambulanten Bereich fokussiert. Unterschiedlich gestaltete Demenznetzwerke sollen die ambulante Versorgung effizienter und hochwertiger gestalten. Wolf-Ostermann et al. nutzen die in diesen Netzwerken erhobenen Daten für eine Beschreibung der Teilnehmer mit Demenz. Im Literaturvergleich erscheint diese Gruppe repräsentativ. Neu ist hier die Erfassung des Gefühls von Einsamkeit und des Akzeptanzgefühls in der Gesellschaft.

Ferner werden im vorliegenden Schwerpunktheft zwei zielgerichtete Beschäftigungsangebote für Menschen mit Demenz beschrieben. Morgenstern et al. konnten zeigen, dass ein täglich durchgeführtes „Brain-gym“-Übungsprogramm von Pflegeheimbewohnern mit leichter bis mittelgradiger Demenz erfolgreich durchgeführt werden kann. Die Pflegekräfte der Pflegeinrichtungen konnten durch eine eintägige Fortbildungsveranstaltung für die selbstständige Durchführung des Programms geschult werden. Die in der Tendenz positiven
Effekte dieses Programms ermutigen zur Durchführung einer größeren Studie, für deren Fallzahlberechnung die vorliegende Studie eine Basis bilden könnte. In der von Ehret et al. durchgeführten Studie wurde Besuchern von Tagespflegeinrichtungen mit und ohne Demenz eine Tablet-Version des Memory-Spiels angeboten. Teilweise konnten auch individualisierte Varianten mit privaten Bildern erstellt werden. Unter Aspekten der Verhaltensbeobachtung, der kulturellen Spieltheorie, des Zusammenhangs der Sphären Sprache und Spiel, beschreiben die Autoren in einem deduktiv-induktiv-abduktiven Vorgehen die Ergebnisse anhand der Beobachtung der 14 teilnehmenden Probanden. Die Studie zeigte, dass es auch Menschen mit Demenz gelingt, ein neues technisches System $\mathrm{zu}$ bedienen und, dass das Interesse an diesem Spiel erhalten blieb. Die befragten Betreuerinnen in den Einrichtungen haben die Intervention positiv bewertet.

Um eine adäquate Versorgung von Menschen mit Demenz zu gewährleisten, spielt die Identifikation der betroffenen Menschen eine bedeutende Rolle. Die Mini Mental State Examination nach Folstein et al. [12] wird weitläufig als Screeninginstrument verwendet. Eine der verschiedenen Aufgaben des Tests besteht darin, einen Satz freier Wahl zu schreiben, der eine Satzstruktur aufweist und sinnhaft ist. Bewertet wird dieser Satz nur nach formalen Kriterien. Sniatecki et al. untersuchten nun, inwieweit die inhaltliche Färbung des Satzes mit der Lebensqualität von Menschen mit Demenz oder auch mit depressiven Symptomen assoziiert ist. In der klinischen Praxis sollte uns ein negativ gefärbter Satz aufhorchen lassen.

Bei geriatrischen Patienten, die im Rahmen einer somatischen Erkrankung in ein Akutkrankenhaus aufgenommen wurden, erscheint es sinnvoll, den Aufenthalt für eine Demenzdiagnostik zu nutzen, wenn der Verdacht auf eine kognitive Einschränkung besteht. Estrella et al. untersuchten bei geriatrischen Patienten, die einer Demenzdiagnostik zugestimmt hatten, wie zufrieden sie mit der damit verbundenen Diagnostik waren und ob sie bereit wären, diese nochmalig durchführen zu lassen.
Für Menschen mit Demenz stellt ein Krankenhausaufenthalt eine kritische Situation dar. Die Komplikationsrate ist im Vergleich zu geriatrischen Patienten ohne Demenz erhöht; insbesondere imponieren eine erhöhte Sturzgefahr [13] und eine hohe Delirrate bis zu $89 \%$ [14]. Wie sich diese Problematik auf die Krankenhausverweildauer und Kosten auswirkt, untersuchten Motzek et al. in einer retrospektiven Analyse. Patienten mit Demenz wurden mit einer nach Alter und Geschlecht gepaarten Kontrollgruppe hinsichtlich Verweildauer und im DRG-System zugeordneter Entgelte verglichen. Diese Arbeit verdeutlicht die gesellschaftliche Relevanz aller Bestrebungen, die darauf abzielen, einerseits Krankenhausaufenthalte bei Menschen mit Demenz möglichst zu vermeiden und andererseits die stationäre Versorgung zu verbessern, um Komplikationen zu reduzieren. Der Beitrag von Büter et al. liefert einen Überblick über architektonische Maßnahmen, die sich für eine demenzfreundliche Gestaltung von Krankenhausstationen bewährt haben. Die Architektur soll dazu beitragen, die Selbstständigkeit von Patienten mit Demenz während des Aufenthalts zu erhalten, indem sie zu Aktivität und Mobilität angeregt werden. Anderseits soll die Architektur Menschen mit Demenz Halt und Orientierung geben, um ihnen so ein Gefühl von Sicherheit und Geborgenheit zu vermitteln. Eine nach diesen Prinzipien erfolgende räumliche Gestaltung muss jedoch stets auch im Kontext der pflegerischen Routine auf Station und dem organisatorischen Ablauf gesehen werden.

Noch befinden wir uns erst am Anfang in unserem Bestreben, Menschen mit Demenz in ihrem jeweiligen Umfeld angemessen zu begegnen. Diese Aussage gilt sowohl auf kommunaler Ebene als auch in der speziellen Situation des Krankenhauses. Im Rahmen des Förderprogramms der Robert Bosch Stiftung „Menschen mit Demenz im Akutkrankenhaus" wird gegenwärtig deutschlandweit eine Vielzahl unterschiedlicher Projekte unterstützt, um neue erfolgreiche Ansätze identifizieren zu können [15]. Für eine weitsichtige Gestaltung der zukünftigen Krankenhauslandschaft ist die 
Berücksichtigung der durch diese Initiative sowie in anderweitigen Modellen gewonnenen Erkenntnisse unverzichtbar.

Um den Herausforderungen der Versorgung von Menschen mit Demenz zukünftig noch besser gerecht zu werden, wird es in den nächsten Jahren eines noch größeren Engagements aller Beteiligten im Gesundheitswesen und vermehrt auch in anderen Teilen der Gesellschaft bedürfen.

\section{Korrespondenzadresse}

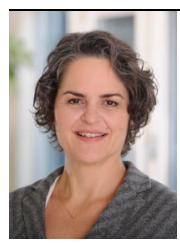

\section{Dr. T. Zieschang}

Agaplesion Bethanien

Krankenhaus

Rohrbacher Str. 149

69126 Heidelberg

Deutschland

tzieschang@

bethanien-heidelberg.de

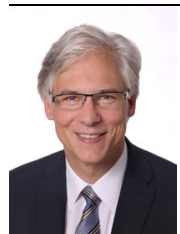

Prof. Dr. J. M. Bauer

Agaplesion Bethanien

Krankenhaus

Rohrbacher Str. 149,

69126 Heidelberg,

Deutschland

juergen.bauer@

bethanien-heidelberg.de

Interessenkonflikt. T. Zieschang und J.M. Bauer

geben an, dass kein Interessenkonflikt besteht.

\section{Literatur}

1. Matthews FE, Arthur A, Barnes LE, Bond J, Jagger C, Robinson L, Brayne C, Medical Research Council Cognitive Function and Ageing Collaboration et al (2013) A two-decade comparison of prevalence of dementia in individuals aged 65 years and older from three geographical areas of England: results of the Cognitive Function and Ageing Study I and II. Lancet 382:1405-1412

2. Langa KM, Larson EB, Crimmins EM, Faul JD, Levine DA, Kabeto MU, Weir DR (2016) A comparison of the prevalence of dementia in the United States in 2000 and 2012. JAMA Intern Med. doi:10.1001/ jamainternmed.2016.6807

3. Eli Lilly and Company (2016) Pressekommunikation von Lilly vom 23.11.2016. http://www. prnewswire.com/news-releases/lilly-announcestop-line-results-of-solanezumab-phase-3clinical-trial-300367976.html. Zugegriffen: 13.12.2016

4. Voss MW, Nagamatsu LS, Lui-Ambrose T, Kramer AF (2011) Exercise, brain, and cognition across the life span.J Appl Physiol 111:1505-1513

5. Lautenschläger NT, Cox KL, Flicker L, Foster JK, van Bockxmeer FM, Xiao J, Greenop KR, Almeida OP (2008) Effect of physical activity on cognitive function in older adults at risk for Alzheimer disease: a randomized trial. JAMA 300:1027-1037

6. DeFina LF, Willis BL, Radford NB, Gao A, Leonard D, Haskell WL, Weiner MF, Berry JD (2013) The association between midlife cardiorespiratory fitness levels and later-life dementia. Ann Intern Med 158:162-168

7. Buchman AS, Boyle PA, Yu L, Shah RC, Wilson RS, Bennett DA (2012) Total daily physical activity and the risk of $A D$ and cognitive decline in older adults. Neurology 78:1323-1329

8. Gates N, Fiatrone Singh MA, Sachdev PS, Valenzuela M (2013) The effect of exercise training on cognitive function in older adults with mild cognitive impairment: a meta-analysis of randomized controlled trails. Am J Geriatr Psychiatry 21:1086-1097

9. Winchester J, Dick MB, Gillen D, Reed B, Miller B, Tinklenberg J, Mungas D, Chui H, Galasko D, Hewett L, Cotman CW (2013) Walking stabilizes cognitive functioning in Alzheimer's disease (AD) across one year. Arch Gerontol Geriatr 56:96-103

10. Ngandu T, Lehtisalo J, Solomon A, Levälahti E, Ahtiluoto $S$, Antikainen R, Bäckman L, Hänninen T, Jula A, Laatikainen T, Lindström J, Mangialasche F, Paajanen T, Pajala S, Peltonen M, Rauramaa R, Stigsdotter-Neely A, Strandberg T, Tuomilehto J, Soininen H, Kivipelto M (2015) A 2 year multidomain intervention of diet, exercise, cognitive training, and vascular risk monitoring versus control to prevent cognitive decline in at-risk elderly people (FINGER): a randomised controlled trial. Lancet 385:2255-2263

11. Hiel L, Beenackers MA, Renders CM, Robroek SJ, Burdorf A, Croezen S (2015) Providing personal informal care to older European adults: should we care about the caregivers' health? Prev Med 70:64-68

12. Folstein MF, Folstein SE, McHugh PR (1975) Minimental state: a practical method for grading the cognitive state of patients for the clinician. J Psychiatr Res 12:189-198

13. Schwendimann R, Bühler H, De Geest $S$, Milisen K (2008) Characteristics of hospital inpatient falls across clinical departments. Gerontology 54:342-348

14. Fick DM, Agostini JV, Inouye SK (2002) Delirium superimposed on dementia: A systematic review. J Am Geriatr Soc 50:1723-1732

15. Robert Bosch Stiftung (2016) Menschen mit Demenzim Akutkrankenhaus. http://www.bosch stiftung.de/content/language1/html/37166.asp. Zugegriffen: 13.12.2016 